\title{
GESTIÓN AMBIENTAL DE RESIDUOS AVÍCOLAS MEDIANTE DIGESTIÓN ANAEROBIA PARA LA PRODUCCIÓN DE FERTILIZANTES ORGÁNICOS LÍQUIDOS
}

\author{
ENVIRONMENTAL WASTE MANAGEMENT OF POULTRY BY ANAEROBIC DIGESTION \\ FOR LIQUIDS ORGANIC FERTILIZER PRODUCTION
}

${ }^{1}$ Fanny Carhuancho L., ${ }^{2}$ Judith Ramírez C. y ${ }^{3}$ Juan Guerrero B.

\begin{abstract}
Resumen
Este trabajo de investigación analizó los efluentes líquidos de procesos de digestión anaerobia de tres tipos de residuos avícolas: Gallinaza de jaula (GJ), Gallinaza de piso (GP) y la mezcla resultante de ambas Gallinaza Mezcla (GM), con la finalidad de obtener un efluente apropiado para uso agronómico. Para ello, se han empleado biodigestores tipo batch en un proceso de fermentación bajo condiciones naturales, sin estimuladores y a temperatura ambiente. A lo largo del proceso de digestión se evaluaron parámetros fisicoquímicos, microbiológicos y se realizaron bioensayos de germinación sobre semillas de maíz. Los efluentes obtenidos (EJ, EP y EM) en los tres tratamientos presentaron un contenido elevado de materia orgánica (139 a 19.6 g/l), Nitrógeno (931 a 1813 mg/l), Potasio (2224-2500 mg/l) y Fósforo (109.77 - $164.76 \mathrm{mg} / \mathrm{l})$ entre otros macro y micro nutrientes. Asimismo, se obtuvieron valores inferiores a $3 \mathrm{NMP} / \mathrm{ml}$ de coliformes totales y fecales, que indican la ausencia de estos microorganismos; por lo que pueden ser usados como fertilizantes orgánicos y constituir una alternativa viable en la gestión de residuos de la actividad avícola.
\end{abstract}

Palabras claves: Residuo avícola; digestión anaerobia, fertilizante orgánico, biofertilizante.

\begin{abstract}
This research analyzed the liquid effluents from anaerobic digestion of three types of poultry waste: Poultry cage $(\mathrm{PC})$, Poultry floor (PF) and the resulting mixture of both Poultry Mix (PM), in order to obtain a suitable effluent for agronomic use. For this, batch-type digesters have been employed in a fermentation process under natural conditions without stimulators at room temperature. During the digestion process physicochemical and microbiological parameters were evaluated and bioassays were performed on seed germination corn. The effluents obtained (EJ, EP and EM) in the three treatments had a high organic matter content (139 to $19.6 \mathrm{~g} / 1)$, nitrogen (931-1813 mg / 1), potassium (2224-2500 $\mathrm{mg} / \mathrm{l}$ ) and Phosphorus (109.77 - $164.76 \mathrm{mg} / \mathrm{l}$ ) among other macro and micro nutrients. Also, values below $3 \mathrm{MPN} / \mathrm{ml}$ of total coliforms and fecal values, indicating the absence of these microorganisms were obtained; so they can be used as an organic fertilizer and a viable alternative in the management of waste from the poultry industry.
\end{abstract}

Key words: poultry manure;Anaerobic digestion, organic fertiliser, biofertiliser.

\section{Introducción}

La producción avícola se ha convertido en una actividad intensiva que representa el $54 \%$ del subsector pecuario a nivel nacional (MINAG, 2010), agrupándose en la costa peruana debido a que las condiciones climáticas son favorables para la crianza de aves. La concentración de aves de corral puede presentar problemas para la disposición y gestión de los residuos. A la fracción orgánica de estos se le conoce como gallinaza y se compone de heces, orina, porción no digerible de alimentos, microorganismos de la biota intestinal, plumas y huevos rotos de la gallina criada en jaula o piso (Estrada, 2005).

Las alternativas para la gestión de estos residuos pueden ser el compostaje, su uso como alimento balanceado, combustión directa y digestión anaerobia. Sin embargo, el compostaje se ve limitado debido a razones de espacio para la producción del compost, según Neal, (2013), por la emisión de amonio al ambiente según Elwell et al, (1998) y por la contaminación de agua de escorrentía por fósforo según Dávalos et al (2002). El uso como alimento balanceado señalado por Ríos de Álvarez et al, (2005), Villarreal, (2011), Tobia y Vargas, (2009) no está contemplado en la normativa nacional como alternativa viable. Otra opción es la combustión directa para la producción combinada de calor y potencia, que tiene la desventaja de producir gases como el $\mathrm{NO}_{\mathrm{x}}$ y $\mathrm{SO}_{2}$ (Williams, 1999).

\footnotetext{
${ }^{1}$ Universidad Nacional Agraria La Molina. Lima, Perú. E-mail: mabel.carhuancho@gmail.com

${ }^{2}$ Universidad Nacional Agraria La Molina. Lima, Perú. E-mail: jramirez@lamolina.edu.pe

${ }^{3}$ Universidad Nacional Agraria La Molina. Lima, Perú. E-mail: jguerrerob@lamolina.edu.pe
} 
Estudios de Kelleher et al, (2002), Krylova et al, (1997), Itodo et al.(1997), Magbanua et al (2001) y Bujoczek et al, (2000), han documentado que es factible el uso de la fracción biodegradable de los residuos de la industria avícola en procesos de digestión anaerobia como una alternativa para la generación de energía y el uso del lodo residual como fertilizante para cultivos ha sido estudiado por Neal, (2013), Avibiol, (2010). La posibilidad de utilizar gallinaza como un único sustrato para un proceso anaeróbico fue probado por Abouelenien et al, (2009), mientras que Szogi y Vanotti, (2009) encontraron que se pueden obtener más beneficios económicos si se utilizan subproductos de la transformación del estiércol de aves, que vendiéndolos directamente. Un fertilizante alternativo, como el fertilizante orgánico obtenido a partir de efluente de un digestor anaeróbico, podría reducir la necesidad de fertilizantes a base de amoníaco y disminuir la energía utilizada en el proceso de producción de fertilizante sintético (Neal, 2013).

Por esta razón, se considera importante evaluar un proceso de digestión anaerobia a partir de gallinaza y bajo condiciones naturales, lo que implica una fermentación a temperatura ambiental y sin aplicación de inóculos o microorganismos eficientes para la obtención de fertilizante orgánico, a fin de demostrar la aplicabilidad de esta tecnología como propuesta de gestión ambiental de los residuos de la actividad avícola.

\section{Materiales y métodos}

Muestra de Residuos: La toma de muestra de gallinaza se ha realizado mediante la metodología asistemática (muestreo al azar) de los galpones de crianza de gallinas en jaula y piso de la granja de aves de la Facultad de Zootecnia, Universidad Nacional Agraria La Molina (UNALM). Fueron llevadas al laboratorio en bolsas cerradas de un kilo cada una. Estas muestran en adelante se denominarán: Estiércol de gallina en piso (Gallinaza piso - GP), estiércol de gallina en jaula (Gallinaza Jaula GJ) y estiércol de gallina en piso + estiércol de gallina en jaula (Gallinaza Mezcla- GM). Se registró la información del tipo de alimentación, edad, especie y manejo de los residuos de las aves.

Preparación de las muestras y formulación de la carga del digestor: Para cada muestra se ha determinado su porcentaje de humedad, composición, peso e impurezas por tamizado y se ha analizado fisicoquímica y microbiológicamente. Se ha determinado la relación carbono nitrógeno, considerando que una relación $\mathrm{C} / \mathrm{N}$ óptima es 30, según Soria et al, (2000). Se ha determinado asimismo, los sólidos totales de cada muestra mediante el método 2540B del Standard methods of examination of water and wastewater (APHA, 2006). Las diluciones (peso de materia seca y peso de materia húmeda) para la carga de cada sistema se han elaborado según Restrepo (2001), seleccionado la relación 1:4.
Digestión de las muestras: El sistema de digestión utilizado ha consistido en contenedores de 81 de capacidad, sellados herméticamente, con conexiones para monitoreo de temperatura y $\mathrm{pH}$. El diseño experimental ha consistido en 2 repeticiones por muestra haciendo un total de 6 prototipos. Una vez finalizado el proceso, se seleccionaron 3 muestras de efluente resultante de los mejores tratamientos, para realizar análisis fisicoquímico y microbiológico.

Monitoreo del sistema: Se ha controlado las variables $\mathrm{pH}$ y temperatura de las fases sólida (parte de la mezcla con menos contenido de agua y suspendido) y líquida (parte de la mezcla con menos contenido de sólido). Se han utilizado termistores para el control de la temperatura y su evaluación ha sido diaria. Una válvula se ha conectado al digestor para extraer muestras y evaluar $\mathrm{pH}$ cuatro veces por semana. Las mediciones se han realizado considerando la norma técnica para compost TMECC 4.11-A Test Methods for the Examination of Composting and Compost (USC Composting Council, 2001)

Análisis fisicoquímicos y microbiológicos: Se han realizado al inicio y al final de las pruebas de digestión anaerobia. Al inicio las muestras analizadas fueron el estiércol de gallina (GP, GJ y GM). Se ha analizado el contenido de NPK, pH, Conductividad eléctrica, materia orgánica, sólidos totales, humedad, carbono orgánico, nitrógeno y relación $\mathrm{C} / \mathrm{N}$. Al finalizar la prueba se analizaron los efluentes (EP, EJ y EM), en cuanto a su cantidad macro nutrientes ( $N, P, K, C a, M g$ y $\mathrm{Na}$ ), micronutrientes ( $\mathrm{Fe}, \mathrm{Cu}, \mathrm{Zn}, \mathrm{Mn}$ y B), $\mathrm{pH}$, conductividad eléctrica, sólidos totales y materia orgánica. Los análisis fisicoquímicos y contenidos de nutrientes se han realizado en el Laboratorio de Análisis de Suelos, Plantas, Aguas y fertilizantes (LASPAF) de la UNALM. Los parámetros microbiológicos analizados han sido Coliformes totales y fecales y se realizaron en el laboratorio de Ecología Microbiana y Biotecnología Marino Tabusso-UNALM.

Prueba de toxicidad y bioensayo de germinación en placas: La prueba de toxicidad se ha llevado a cabo mediante el método de test de Sobrero y Ronco, (2004) adaptado para efluente líquido. El test se ha aplicado sobre los mejores tratamientos. Para el bioensayo de germinación en placas se ha utilizado un volumen muestral de $1 \mathrm{ml}$ de efluente de cada biodigestor con diluciones por cada muestra, utilizando 10 semillas de maíz (Zea mays) por placa tomando como referencia las diluciones planteadas por Peralta (2010). Se ha evaluado el porcentaje de germinación y longitud de la radícula con el fin de obtener el índice de germinación.

Análisis Estadístico: Se ha realizado un análisis de varianza (ANOVA) mediante la prueba $\mathrm{T}$ Student al $95 \%$ de confiabilidad y la distribución normal al 99\% de 
confiabilidad para las variables de $\mathrm{pH}$ y temperatura de los tres tratamientos. La prueba de toxicidad y bioensayo se realizo en base a un análisis de Varianza (p 0.05) para un diseño completamente al azar con tres repeticiones.

\section{Resultados y discusión}

\section{Composición de las muestras}

La composición de las muestras analizadas se presenta en la tabla 1. Por el tipo de crianza y el manejo de las aves en la granja UNALM, se observa porcentajes en el rango de 15.2 a 22.6 de materiales orgánicos (residuos de madera y animales detritívoros) que pueden presentar dificultad en el proceso de digestión debido a su composición.

Tabla 1. Composición de estiércol de gallina en piso y jaula.

\begin{tabular}{cccc}
\hline $\begin{array}{c}\text { Estiércol } \\
\text { fresco } \\
\text { en piso }\end{array}$ & $\begin{array}{c}\text { Porcentaje } \\
\%\end{array}$ & $\begin{array}{c}\text { Estiércol fresco } \\
\text { en jaula }\end{array}$ & $\begin{array}{c}\text { Porcentaje } \\
\%\end{array}$ \\
\hline Excreta & 76.6 & Excreta & 82.7 \\
Plumas & 0.2 & Plumas & 0.3 \\
$\begin{array}{c}\text { Material } \\
\text { absorbente* } \\
\text { Otros }\end{array}$ & 22.6 & $\begin{array}{c}\text { Animales } \\
\text { detritívoros. }\end{array}$ & 15.2 \\
residuos & 0.6 & Otros residuos & 0.8 \\
*viruta: residuo de madera aserrada
\end{tabular}

\section{Caracterización fisicoquímica y microbiológica de las muestras}

El resultado del análisis de parámetros fisicoquímicos de GJ, GP y GM se aprecia en la tabla 2.

Tabla 2. Análisis fisicoquímicos de muestras de Gallinaza.

\begin{tabular}{lccc}
\hline Parámetros & GJ & GP & GM \\
\hline pH & 6.45 & 6.38 & 6.21 \\
Conductividad $(\mathrm{dS} / \mathrm{m})$ & 18.30 & 14.60 & 16.40 \\
Humedad $(\%)$ & 60.88 & 26.08 & 40.30 \\
Solidos totales $(\%)$ & 31.37 & 93.12 & 59.41 \\
Materia orgánica $(\%)$ & 54.66 & 66.72 & 61.23 \\
Carbono orgánico $(\%)$ & 31.71 & 38.70 & 35.52 \\
Nitrógeno $(\%)$ & 3.24 & 3.37 & 3.23 \\
Relación $\mathrm{C} / \mathrm{N}$ & 9.79 & 11.48 & 11.00 \\
Potasio $\left(\mathrm{K}_{2} \mathrm{O} \%\right)$ & 3.14 & 2.89 & 2.58 \\
Fósforo $\left(\mathrm{P}_{2} \mathrm{O}_{5} \%\right)$ & 4.77 & 4.94 & 4.85 \\
\hline
\end{tabular}

El pH de los tres tipos de gallinazas varían entre 6.2 a 6.5 en la escala ligeramente acida, presentando la muestra de gallinaza en jaula un valor superior a las otras dos. La variación se debe a la composición del estiércol debido principalmente a su manejo.

El contenido de coliformes fecales y totales de las muestras se aprecian en la tabla 3 .
Tabla 3. Análisis microbiológico de muestras de Gallinaza.

\begin{tabular}{cccc}
\hline Parámetros & GJ & GP & GM \\
\hline Coliformes totales (NMP/g) & $11 \times 10^{6}$ & $11 \times 10^{6}$ & $70 \times 10^{6}$ \\
Coliformes fecales (NMP/g) & $11 \times 10^{6}$ & $11 \times 10^{6}$ & $70 \times 10^{6}$ \\
\hline
\end{tabular}

De acuerdo a la Agencia de Protección del Medio Ambiente de los Estados Unidos (EPA por sus siglas en ingles), los límites máximos permitidos de contenido de coliformes fecales en los productos orgánicos a ser utilizados como fertilizantes se aprecian en el Tabla 4.

Tabla 4.Límites máximos permitidos de contenidos de coliformes fecales en productos orgánicos.

\begin{tabular}{ccc}
\hline Clase & Cultivos & $\begin{array}{c}\text { Limite NMP/g } \\
\text { MS }\end{array}$ \\
\hline Clase A & $\begin{array}{c}\text { Cultivos de consumo directo } \\
\text { Cultivos de consumo no } \\
\text { directo }\end{array}$ & $<1.000$ \\
Clase B & $<2 \times 10^{6}$ \\
\hline Fuente: EPA (1999) &
\end{tabular}

Los valores de coliformes totales y fecales que se han obtenido, limitan la utilización de la gallinaza cruda sin tratamiento. Basados en los criterios de la EPA, la fertilización directa no sería recomendable debido al riesgo sanitario de contaminación de los cultivos y el suelo.

\section{Carga del Biodigestor}

Según los resultados fisicoquímicos de los tres tipos de estiércol se calculó la relación $\mathrm{C} / \mathrm{N}$ y \% de sólidos totales como se aprecia en la tabla 5. La relación $\mathrm{C} / \mathrm{N}$ es menor al valor recomendado por Soria et al (2000), por lo cual se mezcló la gallinaza con rastrojo de maíz para elevar la relación $\mathrm{C} / \mathrm{N}$ a 30 .

Tabla 5. Relación C/N y \% Sólidos Totales.

\begin{tabular}{lcccc}
\hline & $\% \mathrm{C}$ & $\% \mathrm{~N}$ & $\mathrm{C} / \mathrm{N}$ & Sólidos totales \% \\
\hline GJ & 31.7 & 3.2 & 9.8 & 31.4 \\
GP & 38.7 & 3.4 & 11.5 & 93.1 \\
GM & 35.5 & 3.2 & 11.0 & 59.4 \\
Chala & 51.9 & 0.9 & 61.8 & 90.8 \\
\hline
\end{tabular}

\section{Monitoreo del funcionamiento del Biodigestor}

Monitoreo del $\mathrm{pH}$

La variación de $\mathrm{pH}$ se observa en la figura 1, durante los primeros quince días el valor fue inestable, con una variación de 7.3 a 5.1; a partir del quinceavo día se observa menor variabilidad de los datos. El pH promedio al finalizar el proceso varía entre 5.2 a 5.4 . 


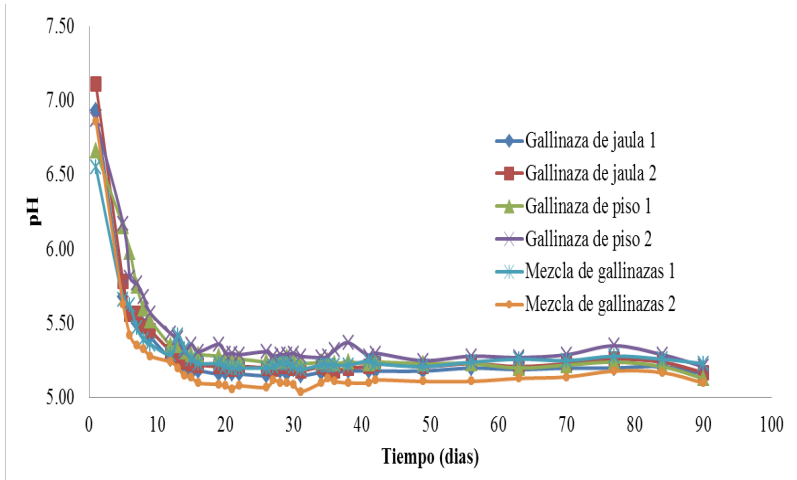

Figura 1. Variación de $\mathrm{pH}$ de los tratamientos con Gallinaza.

Según el análisis de varianza no se presentaron diferencias significativas de $\mathrm{p}$

$\mathrm{H}$ entre los 3 tratamientos, con una confiabilidad de 99\%.

Según Sung y Liu, (2003) citado por Rivera (2010), el $\mathrm{pH}$ determina la composición del nitrógeno amoniacal total donde a bajos niveles de $\mathrm{pH}$ predomina el amonio, además las pequeñas disminuciones de $\mathrm{pH}$ implican el consumo elevado de alcalinidad disminuyendo la capacidad amortiguadora del $\mathrm{pH}$ del medio, por ende, la capacidad de neutralizar los ácidos grasos es baja. Pelczar y Reid, (1958) citado por García, et al., (2005), mencionan que la disminución del pH según el tiempo de fermentación puede ser consecuencia de la producción de ácidos grasos de cadenas cortas, que inhiben o eliminan los microorganismos que crecen a $\mathrm{pH}$ más neutros, por lo cual se deduce que el proceso no llego a la fase acetogénica ni metanogénica donde el $\mathrm{pH}$ varían entre 6.5 y 7.5 (Martí, 2006).

\section{Monitoreo de la temperatura durante el proceso}

La variación de la temperatura interna del Biodigestor, en todos los tratamientos ha sido muy inestable, presentando incremento y disminución durante todo el proceso. La temperatura de las fases líquida y sólida coincide con un descenso a partir del día 15 , los mismos que concuerdan con la estabilización del $\mathrm{pH}$ para la misma época.

En la figura 2 se observa la variación de la temperatura de la fase líquida y sólida del tratamiento GJ. Al quinceavo día la muestra presenta un descenso de la temperatura estabilizándose en los últimos días del proceso con un valor mayor a $19^{\circ} \mathrm{C}$. El rango de temperatura se encuentra en el mesofilico.

En la figura 3, se observa la variación de temperatura para los tratamientos GP, el cual presenta mayor temperatura que los tratamientos para GJ debido a su composición fisicoquímica, el rango de temperatura se encuentra en mesofilico. En la figura 4, se observa la variación de temperatura de los tratamientos GM. La temperatura de la fase liquida es mayor a la temperatura de la fase sólida. Se ha estabilizado al final del proceso en un promedio de $18^{\circ} \mathrm{C}$, encontrándose en la fase psicrofilica

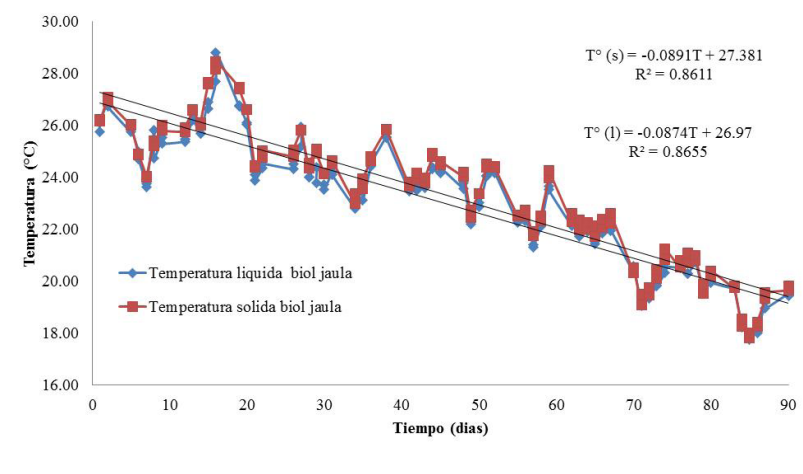

Figura 2. Análisis de regresión lineal del tratamiento de gallinaza de jaula -GJ.

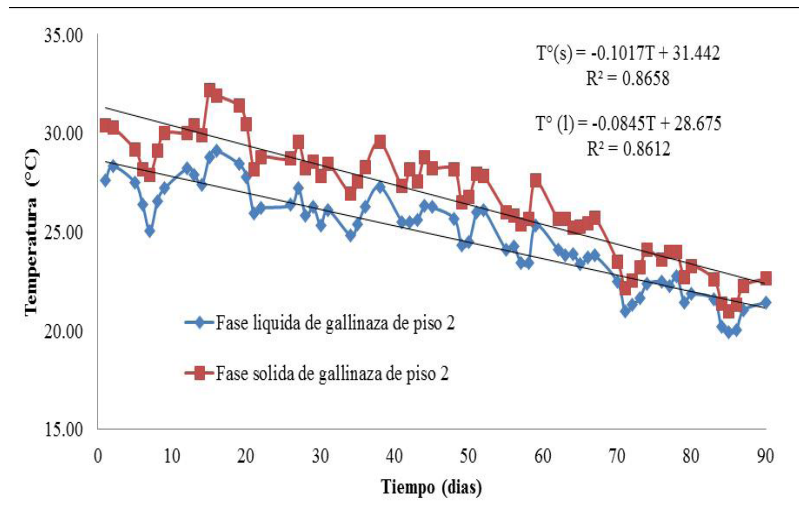

Figura 3. Análisis de regresión lineal del tratamiento gallinaza en piso- GP.

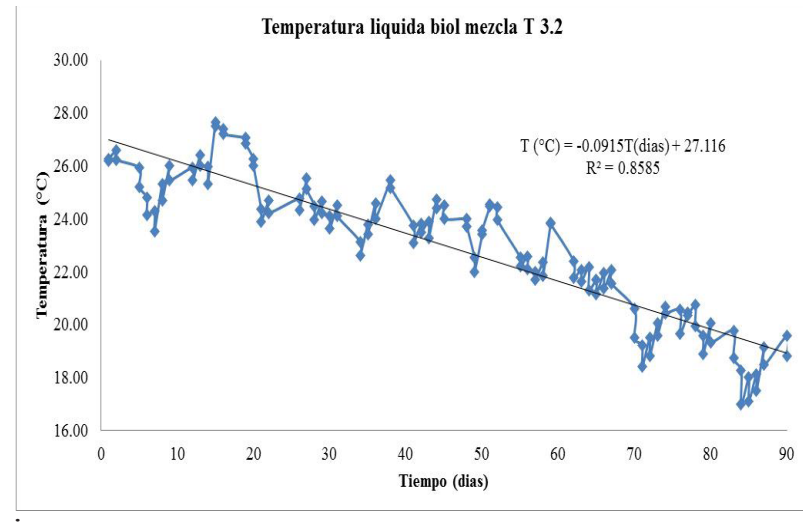

Figura 4. Análisis de regresión lineal del tratamiento de mezcla de gallinazas - GM.

Según el análisis de varianza las temperaturas líquidas y solidas en las dos repeticiones de los tres tratamientos no presentaron diferencias significativas con una confiabilidad de 99\%. Mediante análisis de la regresión lineal, el coeficiente de determinación obtenido indica que el $86.58 \%$ y $86.12 \%$ de la variabilidad de la temperatura de las fases sólida y líquida es explicado por el tiempo de retención respectivamente, lo que indica que la variable temperatura es estocástica debido a que su variabilidad no se puede controlar y va depender de condiciones climáticas 
Como se observa en las tres figuras anteriores, la etapa mesofílica en los cuatro tratamientos ha durado 60 días, luego ha iniciado la etapa psicrofilica ascendiendo a partir del día 85 y volviendo a la etapa mesofílica al final del proceso, excepto en los tratamientos de gallinaza de mezcla - GM.

A lo largo del proceso de digestión se observó la disminución de $\mathrm{pH}$ y temperatura, como consecuencia del proceso natural de digestión anaerobia.

\section{Caracterización del efluente líquido obtenido Análisis microbiológico}

Se han realizado análisis para verificar la ausencia de microrganismos patógenos como coliformes fecales y totales, los cuales puedan afectar la inocuidad del abono foliar en la pruebas de bioensayos en maíz. En la tabla 6 se muestra los resultados de los análisis

Tabla 6. Análisis microbiológico de efluentes de los tres tipos de gallinaza.

\begin{tabular}{lcccc}
\hline & $\begin{array}{c}\text { Gallinaza } \\
\text { fresca }\end{array}$ & EJ & EP & EM \\
\hline $\begin{array}{l}\text { Enumeración de coliformes } \\
\text { totales NMP/ml }\end{array}$ & $10^{6} \mathrm{NMP} / \mathrm{g}$ & $<3$ & $<3$ & $<3$ \\
$\begin{array}{l}\text { Enumeración de coliformes } \\
\text { fecales NMP/ml }\end{array}$ & $10^{6} \mathrm{NMP} / \mathrm{g}$ & $<3$ & $<3$ & $<3$ \\
$\begin{array}{l}\text { Nota: Valores de }<3 \text { indican ausencia de microrganismos. } \\
\text { Donde: } \mathbf{E J}: \text { Efluente jaula; } \mathbf{E P}: \text { Efluente piso; EM: Efluente mezcla }\end{array}$
\end{tabular}

Los resultados de los análisis muestran la ausencia de coliformes fecales y totales en los tres tratamientos dando valores $<3 \mathrm{NMP} / \mathrm{ml}$ luego de un tiempo de 90 días de fermentación. Este valor indica la ausencia de coliformes totales y fecales en el abono foliar.

Se verifica la disminución de la carga microbiana inicial. Comparando los valores obtenidos con el Estándar de Calidad Ambiental para Agua (D.S. 002-2008-MINAM) para uso de agua con fines de riego, los microorganismos patógenos deben tener como máximo $1000 \mathrm{NMP} / 100 \mathrm{ml}$ de coliformes fecales y $5000 \mathrm{NMP} / 100 \mathrm{ml}$ de coliformes totales, estando los tratamientos por debajo del límite establecido.

Respecto a la presencia de otros microrganismos como bacterias totales, bacterias Gram+, Gram-, estafilococos y E. coli, no se han analizado en laboratorio, pero según Terzich et al. (2000) citado por Garcia et al. (2005), el número de estas bacterias en el estiércol tienden a incrementarse con el ascenso del pH. En el proceso de digestión se observó un descenso del $\mathrm{pH}$ por lo cual teóricamente no existe riesgo de estos patógenos en el Efluente.

Análisis químico de interés agronómico del efluente de digestión anaerobia de la gallinaza

En la tabla 7 se muestra el resultado del análisis fisicoquímico y contenido de macro y micronutrientes de los efluentes con el objetivo de evaluar el potencial que tiene este producto como abono orgánico líquido.

El efluente de gallinaza en jaula y piso contiene una alta concentración de nitrógeno valores de $1708 \mathrm{mg} / 1$ y $1813 \mathrm{mg} / \mathrm{l}$ respectivamente, esto se debe que el nitrógeno contenido en la gallinaza no se ha perdido debido al $\mathrm{pH}$ bajo $>5$.

Tabla 7. Análisis fisicoquímico y contenido de macro y micronutrientes de los efluentes EJ, EP y EM.

\begin{tabular}{|c|c|c|c|}
\hline Parámetros & EJ & $\mathrm{EP}$ & EM \\
\hline $\mathrm{PH}$ & 5.07 & 5.08 & 4.97 \\
\hline $\mathrm{CE}(\mathrm{dS} / \mathrm{m})$ & 20.2 & 20.6 & 16.3 \\
\hline Solidos totales & 30.8 & 35 & 25.1 \\
\hline $\mathrm{MO}(\mathrm{g} / \mathrm{l})$ & 18.3 & 19.6 & 13.9 \\
\hline \multicolumn{4}{|c|}{ Análisis de macronutrientes } \\
\hline $\mathrm{N}$ total $(\mathrm{mg} / \mathrm{l})$ & 1708 & 1813 & 931 \\
\hline$P$ total $(\mathrm{mg} / \mathrm{l})$ & 142.08 & 164.76 & 109.77 \\
\hline $\mathrm{K}$ total $(\mathrm{mg} / \mathrm{l})$ & 2300 & 2500 & 2224 \\
\hline Ca total $(\mathrm{mg} / \mathrm{l})$ & 1916 & 2534 & 1968 \\
\hline $\mathrm{Mg}$ total $(\mathrm{mg} / \mathrm{l})$ & 408 & 460 & 336 \\
\hline $\mathrm{Na}$ total $(\mathrm{mg} / \mathrm{l})$ & 332 & 392 & 312 \\
\hline \multicolumn{4}{|c|}{ Análisis de micronutrientes } \\
\hline Fe total $(\mathrm{mg} / \mathrm{l})$ & 82.40 & 101 & 62.4 \\
\hline $\mathrm{Cu}$ total $(\mathrm{mg} / \mathrm{l})$ & 0.64 & 0.82 & 0.32 \\
\hline $\mathrm{Zn}$ total $(\mathrm{mg} / \mathrm{l})$ & 3.64 & 3.92 & 2.16 \\
\hline Mn total $(\mathrm{mg} / \mathrm{l})$ & 9.84 & 10.7 & 8.96 \\
\hline B total $(\mathrm{mg} / \mathrm{l})$ & 3.92 & 5.72 & 4.67 \\
\hline
\end{tabular}

Los tres tipos de efluentes obtenidos EP, EJ, EM a condiciones naturales presentan buena concentración de nitrógeno, potasio y fósforo, además una buena concentración de materia orgánica que es una propiedad importante en los abonos orgánicos como fuente de nutrientes para la microflora del suelo así como un buen medio de soporte para los cultivos.

La muestra EP contiene mayor concentración de nitrógeno debido que la disminución de $\mathrm{pH}$ en comparación al $\mathrm{pH}$ inicial (estiércol fresco) fue menor en gallinaza de piso (GP). Comparando los tres tratamientos seleccionados, el orden respecto a la concentración de nitrógeno es de la siguiente manera EP $>$ EJ $>$ EM. La muestra EP presentó mayor concentración de micro y macro nutrientes especialmente nitrógeno, esto se debe a las condiciones iniciales de la muestra y al manejo del estiércol, la muestra pierde poco nitrógeno al ser expuesto al aire libre y durante el proceso de digestión presenta menor valor de $\mathrm{pH}$.

La tabla 8 muestra una comparación de la composición de la muestra EP con la de otros abonos orgánicos líquidos de estudios de características similares. 
Tabla 8. Análisis macronutrientes del Efluente de diferentes insumos.

\begin{tabular}{|c|c|c|c|c|c|}
\hline Ensayos & $\mathrm{BC}_{(1)}$ & $\mathrm{BV}_{(2)}$ & $\mathrm{BCa}_{(3)}$ & FB $20_{(4)}$ & EP \\
\hline $\mathrm{PH}$ & 7.8 & 7.89 & 7.2 & 3.75 & 5.08 \\
\hline $\mathrm{CE}(\mathrm{dS} / \mathrm{m})$ & --- & 19.28 & 21.3 & 25.70 & 20.6 \\
\hline M.O. en solución g/l & --- & 5.28 & 17.2 & 181.10 & 19.6 \\
\hline Nitrógeno mg/l & 900 & 1876 & 1700 & 4200 & 1813 \\
\hline Fosforo mg/l & 120 & 71.2 & 3800 & 744.20 & 164.76 \\
\hline Potasio mg/1 & 900 & 1940 & 5200 & 17200 & 2500 \\
\hline
\end{tabular}

aves La Calera. Efluente origen gallinaza; (4) Fast Biosol 20 de origen vacuno, Peralta (2010).

La concentración de los principales macronutrientes $\mathrm{N}$, $\mathrm{P}$ y $\mathrm{K}$ de Efluente piso han sido superiores comparado a los bioles de origen porcino y de estiércol de cuy. La diferencia de la concentración de nutrientes se debe principalmente al $\mathrm{pH}$ neutro que obtienen.

Sin embargo, el Fastbiol 20 obtenido en 30 días con un precompost a $85^{\circ} \mathrm{C}$ logra concentraciones superiores a la muestra EP, el nitrógeno es superior debido que el fastbiol se mantiene a un $\mathrm{pH}$ bajo 3.75 , gracias al ácido láctico que evita la perdida de nitrógeno contenido en la materia prima.

Adicionalmente la muestra EP, se compara con el efluente (Biol) obtenido de El Fundo La Calera, que utiliza un sistema de digestión semicontinuo a una temperatura de $38^{\circ} \mathrm{C}$, con agitación constante y contenido de materia seca de $11 \%$. Se observa que los valores de concentración de nitrógeno son menores que los de EP.

\section{Pruebas de Toxicidad y Bioensayo de germinación}

La calidad agronómica del efluente de los diferentes tipos de muestras se ha evaluado mediante bioensayos de germinación con semillas de maíz (Test de Sobrero y Ronco, 2004). Se ha medido el índice de germinación (IG) y la longitud de radícula de semillas de maíz. Se tomó como referencia la prueba presuntiva de la dosis de inhibición media de Peralta (2010), considerando siete dosis de estos materiales (efluente por muestra/agua): 0.1/100, 1/100, 5/100, 7.5/100, 8.8/100, 10/100 y 50/100. Un control sin adición también fue incluido.

En la figura 5 se observa el porcentaje de semillas de maíz germinadas al quinto día para cada tipo de efluente y para la dilución control. Las diluciones cuyos porcentajes de germinación son superiores al $90 \%$ son $0.1 / 100,1 / 100$ y 5/100, de los tres tipos de efluentes. La muestra EM germina además con las diluciones 7.5/100 y 8.8/100. En la dilución 50/100 de las muestras EP y EJ no hay desarrollo de germinación debido al efecto toxico del efluente.

El porcentaje de crecimiento de la radícula (Figura 6), muestra que la elongacion de la dilución control en promedio es de $26.33 \mathrm{~mm}$. Las dosis cuyas elongaciones son mayores a este valor son las diluciones $0.1 / 100$ y 1/100 de la muestra EP y 1/100 de la muestra EM. Según el análisis estadistico, no hay relación entre la elongación de las semillas germinadas y el incremento de las dosis en las diluciones de las muestras EP y EM.

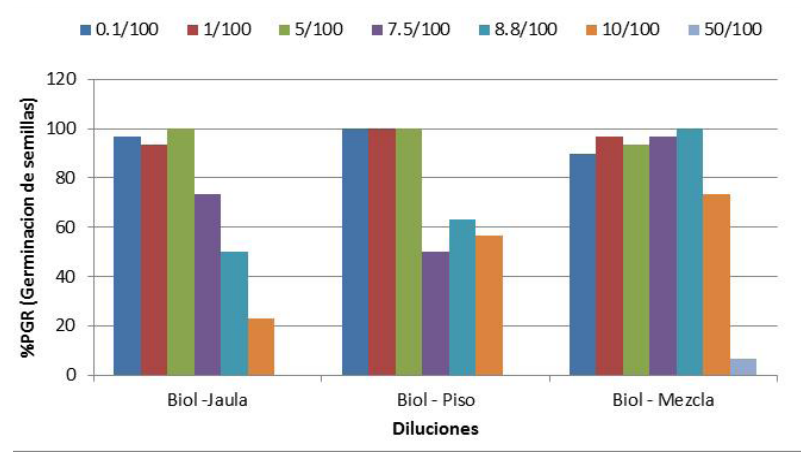

Figura 5. Porcentaje de semillas de maíz germinadas de los tres tipos de efluentes al día cinco.

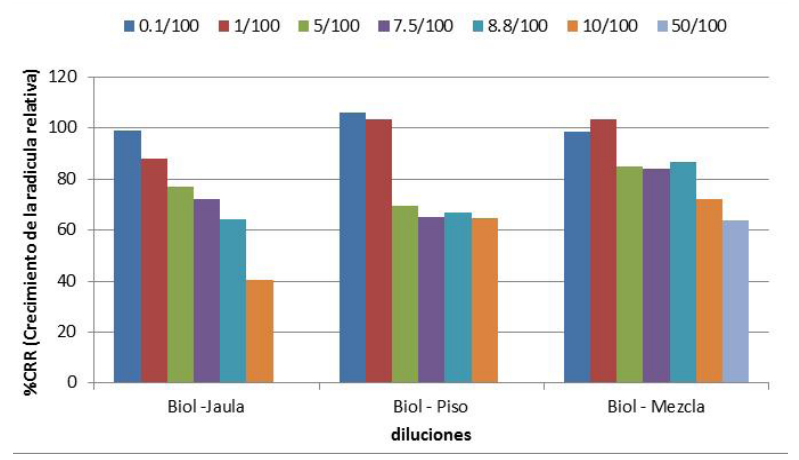

Figura 6. Porcentaje de crecimiento de la radícula por efecto de la aplicación de los tres tipos de efluentes.

Índice de Germinación.

Los IG, en las diluciones $0.1 / 100$ y $1 / 100$ de los tres tipos de muestras y las diluciones 7.5/100 y 8.8/100 de EM, han sido superiores al $80 \%$ (Figura 7). Según Zucconi et al, (1981) citado por Varnero et al., (2007) estos valores indican concentraciones muy bajas o ausencia de sustancias toxicas. La dilución 0.1/100 y 1/100 de la muestra EP supera el valor de $100 \%$, esto puede ser considerado como fitonutriente o fitoestimulante. 


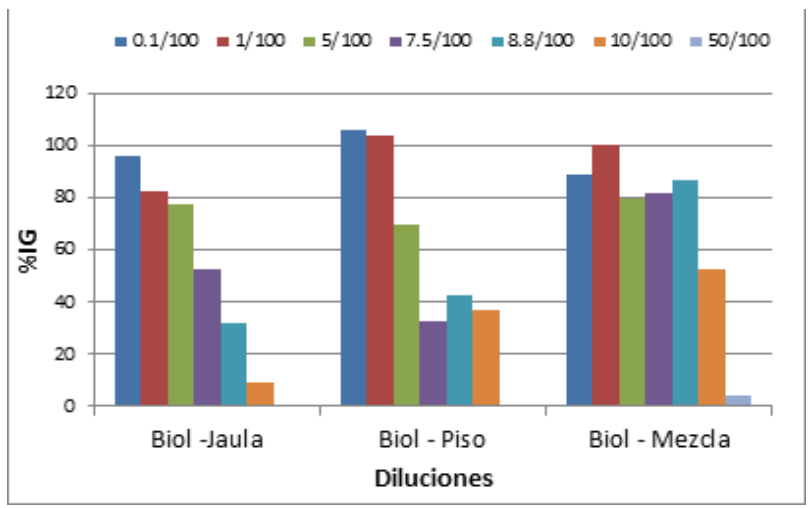

Figura 7. Índice de germinación de los tres tipos de Efluente a las 120 horas

Los rangos de IG $80 \%$ a $50 \%$ indican presencia moderada de sustancias fitotoxicas debido a las altas concentraciones de producto, conjuntamente relacionado con el pH ligeramente ácido que afecta la absorción de los nutrientes de las plántulas según Kupper et al (2002) citado por Peralta, (2010). En los tres tratamientos, las diluciones que resultan ser menores al 50\%, indican que la concentración de nutrientes y las altas concentraciones de sales impiden el crecimiento normal de las plántulas.

\section{Conclusiones}

Las mediciones de los sistemas de digestión analizados, demostraron que sin ningún pre tratamiento del residuo gallinaza, a los 90 días se logró obtener un efluente con importantes aportes de nutrientes en valores de macro, micronutrientes y materia orgánica comparativamente con otros efluentes estudiados. Asimismo, se redujo el contenido de coliformes fecales y totales.

Sobre los tres efluentes analizados (EP, EG, EM) no se encontraron diferencias significativas con respecto a sus características fisicoquímicas, microbiológicas y contenido de macro y micronutrientes. La cantidad de coliformes fecales y totales cumplen con los estándares para calidad de agua de riego y para uso como fertilizante según el MINAM y EPA respectivamente.

Con el análisis de germinación de semilla se demostró que los efluentes resultantes poseen un IG superior al $80 \%$ para diluciones $0.1 / 100$ y $1 / 100$, por lo cual no presentan riesgos de toxicidad en su uso, especialmente el fluente EP. De acuerdo al análisis de varianza, el efluente EM tiene un IG superior a un rango mayor de dosis, por lo que podría tener mayor aplicación foliar en cultivos.

La digestión anaerobia del residuo avícola gallinaza, bajo condiciones naturales, puede obtener un efluente de buena calidad agronómica. Esta técnica podría constituirse entonces, en una alternativa al manejo de los residuos orgánicos de la actividad avícola.

\section{Literatura citada}

Abouelenien, F.; Nakashimada, Y. and Nishio, N. 2009. Dry mesophilic fermentation of chicken manure for production of methane by repeated batch culture. Jouranl of bioscience and bioengineering 107(3): 293-295 p.

Agencia de Protección del Medio Ambiente de los Estados Unidos (EPA por sus siglas en inglés). 1999. Standards for the use or disposal of sewage sludge. 40 CRF part 503. Biosolids Rule. Washington, EUA.

APHA (American Public Health Association). 2006. 2540 Solids. Standard methods of examination of water and wastewater.

Avibiol, 2013. Avibiol, Enmienda Orgánica. Consultado 10 junio 2013. Disponible en http://www.avibiol.com/

Bujoczek, G.; Oleszkiewicz, J., Sparling, R. y Cenkowski, S. 2000. J. agric. Engng Res. 76: 51- 60. Disponible en http://www.idealibrary.com.

Dávalos, J.; Roux. M. y Jimenez, P. 2002. Evaluation of poultry litter as a feasible fuel. Thermochimica Acta 394: 261-266 p

Elwell, D.; Keener, M,; Carey, S. y Schlak, P. 1998. Compostingunamended chicken manure. Compost Sci. Utilisation 6: 22-35p.

Estrada, M. 2005. Manejo y procesamiento de la Gallinaza. Revista Lasallista de investigación. Antioquia, Colombia. 43-48pp.

García, Y.; Elías, A. y Herrera, F. 2005. Dinámica microbiana de la fermentación in vitro de las excretas de gallinas ponedoras. Revista Cubana de Ciencia Agrícola, vol. 39, núm. 1:75-79 p.

Itodo, I.; Lucas, E. and Kucha, E. 1997. The effect of using solar energy in the thermophilic digestion of poultry waste. J. Eng. Int. Develop. 3: 15-21p.

Kelleher, B.; Leahy, I.; Henihan, J.; O'Dwyer. T.; Sutton, D. and Leahy, M. 2002. Advances in poultry litter disposal technology - a review. Bioresource Technology 83: 27-36p

Krylova, N.; Khabiboulline, R.; Naumova, R. y Nagle, M. 1997. The influence of ammonium and methods for removal during the anaerobic treatment of poultry manure. J. Chem. Technol.Biotechnol. 70: 99-105p.

Magbanua, B.; Adams, T. and Johnston, P. 2001. Bioresour. Technol. 76: 165-168p.

Martín. 2006. Phosphorusprecipitation in AnarobicDigestionProcess. Boca Raton. Florida, USA. 4-15 p.

MINAG (Ministerio de Agricultura, PE). 2010. Producción Pecuaria e Industria Avícola. Oficina de estudios económicos y estadísticos. 11, $14 \mathrm{p}$.

Neal, J. 2013. Anerobic Digester Effluentes as Fertilizer for Hydroponically Grow Tomatoes. Tesis para optar el título de Ingeniero. Estados Unidos. 22 p.

Peralta, R. 2010. Determinación de parámetros óptimos en la producción de fastbiol usando las excretas del 
ganado lechero del establo de la UNALM. Trabajo de investigación para optar el titulo de Biólogo - UNALM. Lima, Perú. 19-20, 33-38 p.

Restrepo, J. 2001. Elaboración de abonos orgánicos fermentados y biofertilizantes foliares. IICA, Costa Rica. $19,121 \mathrm{p}$

Ríos de Álvarez, L.; Combellas, J. y Álvarez, R. (2005). Revisión Uso de excretas de aves en la alimentación de ovinos. Zootecnia Tropical, 23(2): 183-210 p.

Rivera, V. 2010. Estudio cinético de la digestión anaeróbica termofílica de pollinaza a escala piloto. Tesis de Maestría Ciencias en Bioprocesos. Instituto Politécnico Nacional. La laguna de Ticoman, México. $4-10$ p.

Sobrero, M. y Ronco, A. 2004. Ensayo de toxicidad aguda con semillas de lechuga (Lactuca sativa L.). Disponible en http://www2.inecc.gob.mx/publicaciones/ libros/573/cap4.pdf

Soria, M.; Ferrera-Cerrato, R. y Etchevers, J. 2000. Producción de biofertilizantes mediante biodigestion de excreta líquida de cerdo. Instituto tecnológico agropecuario. México. 354-355 pp.

Szogi, A. y Vanotti, M. 2009. Prospects for phosphorus recovery from poultry litter. Bioresource Technology 100: 5461-5465 p.

Tobia, C. y Vargas, E. 2000. Evaluación de las excretas de pollos de engorde (Pollinaza) en la alimentación animal. Agronomía Costarricense. 24(1):55-62p

USC Composting Council. 2001. TMECC 4.11-A Test Methods for the Examination of Composting and Compost. Disponible en http://compostingcouncil.org/ tmecc/

Varnero, M.; Rojas, C. y Orellana, R. 2007. Índices de fitotoxicidad en residuos orgánicos durante el compostaje. J. Soil Sci. Nutr. 7(1): 28-37p

Villarreal, S. 2011. Elaboración de una Dieta Balanceada utilizando Gallinaza como fuente alternativa de proteína en la alimentación de Tilapia Roja Macho (oreochromis spp.). tesis para optar el título de ingeniero Agroindustrial. Ecuador. Consultado 10 mayo. 2014. Disponible en http://hdl.handle.net/123456789/439

Williams, P. 1999. Waste Treatment and Disposal. Wiley, New York. 